Research Paper

\title{
Impact of JAK2V617F Mutation Burden on Disease Phenotype in Chinese Patients with JAK2V617F-positive Polycythemia Vera (PV) and Essential thrombocythemia (ET)
}

\author{
Shixiang Zhao ${ }^{1,2,3^{*}}$, Xiang Zhang ${ }^{1,2^{*}}$, Yang Xu1,2, Yufeng Feng ${ }^{1,2}$, Wenhong Sheng ${ }^{1,2}$, Jiannong Cen ${ }^{1,2}$, Depei \\ $\mathrm{Wu}^{1,2 \bowtie}$, Yue $\operatorname{Han}^{1,2 \bowtie}$ \\ 1. Department of Hematology, The First Affiliated Hospital of Soochow University, No.188.Shizi Street, Suzhou, 215006, P.R. China. \\ 2. Jiangsu Institute of Hematology, Key Laboratory of Thrombosis and Hemostasis of Ministry of Health, The First Affiliated Hospital of Soochow University, \\ Suzhou, 215006, P.R. China. \\ 3. Department of Hematology, The First People's Hospital of Yunnan Province, No.154.Jinbi Road, Kunming, 650100, P.R China. \\ ${ }^{*}$ Co-first author
}

$\triangle$ Corresponding authors: Yue Han, De-Pei Wu; Jiangsu Institute of Hematology, Key Laboratory of Thrombosis and Hemostasis of Ministry of Health, The First Affiliated Hospital of Soochow University, No.188 Shizi Street, Suzhou 215006, P.R. China. Telephone: 86-0512-67781856 Fax: 86-512-67781850 E-mail: hanyuecat@sina.com, hezsx1220@163.com.

() Ivyspring International Publisher. Reproduction is permitted for personal, noncommercial use, provided that the article is in whole, unmodified, and properly cited. See http://ivyspring.com/terms for terms and conditions.

Received: 2014.09.13; Accepted: 2015.11.26; Published: 2016.01.25

\begin{abstract}
Most patients with polycythemia vera (PV) and half of essential thrombocythemia (ET) possess an activating JAK2V617F mutation. The objective of this study was to better define the effect of JAK2V617F mutant allele burden on clinical phenotypes in Chinese patients, especially thrombosis. By real-time polymerase chain reaction (RT-PCR), the JAK2V617F mutation burden was detected in 170 JAK2V617F-positive patients, including $54 \mathrm{PV}$ and $116 \mathrm{ET}$. The results showed that JAK2V617F allele burden was higher in PV than in ET $(P<0.001)$. Higher percentage of patients had JAK2V617F allele burden over $20 \%$ in PV than in ET (68.5\% VS 26.7\%) $(P<0.001)$. In PV patients, higher JAK2V617F allele burden was observed in female $(P<0.05)$ and leukocytosis patients (WBC above $\left.10 \times 10^{9} / \mathrm{L}\right)(P<0.001)$. Meanwhile, ET patients showed increased JAK2V617F allele burden in the group with higher hemoglobin (HGB above 150g/L) $(P<0.05)$, leukocytosis (WBC above $\left.10 \times 10^{9} / L\right)(P<0.001)$, splenomegaly $(P<0.05)$ and thrombosis $(P<0.05)$. In conclusion, the JAK2V617F mutation allele burden is higher in Chinese patients with PV than ET. In PV patients, JAK2V617F mutation burden had influence on WBC counts. And the clinical characteristics of ET patients, such as WBC counts, hemoglobin level, splenomegaly and thrombosis, were influenced by JAK2V617F mutation burden. Male, high hemoglobin (HGB above $150 \mathrm{~g} / \mathrm{L}$ ), and increased JAK2V617F mutation burden (JAK2V617F allele burden $\geq 16.5 \%$ ) were risks of thrombosis $(P<$ 0.05 ) for ET patients by Logistic Regression.
\end{abstract}

Key words: Essential thrombocythemia; Polycythemia vera; JAK2V671F mutation allele burden; Thrombosis.

\section{Introduction}

Philadelphia chromosome-negative chronic myeloproliferative neoplasms ( $\left.\mathrm{Ph}^{-} \mathrm{MPN}\right)$ are clonal hematopoietic diseases which include polycythemia vera (PV), essential thrombocythemia (ET) and primary myelofibrosis (PMF). A gain-of-function point mutation has been reported in the Janus 2 tyrosine kinase (JAK2) gene, which increases JAK2 kinase activity and has the potential to affect clinical outcomes [1-2]. The JAK2V617F mutation is presented in about $95 \%$ of PV patients, and in approximately $50 \%$ of ET 
and PMF patients [3-4].

Homozygosity of JAK2V617F mutation is due to mitotic recombination [2]. It is rarely observed in patients with ET, whereas about one-third of patients with PV have homozygous JAK2V617F mutation [3-4]. Akada $\mathrm{H}$ et al generated an inducible JAK2V617F knock-in mouse, which evoked all major features of human polycythemia vera [5]. Higher JAK2V617F allele burden in PV than that in ET had been reported in several studies [6-8]. Previous studies have indicated that more than $50 \%$ of JAK2V617F mutation to be homozygous and $50 \%$ was regarded as the cut-off value to analyze the impact of JAK2V617F mutation burden on disease phenotype [8-9]. However, since there were mixed wild type cells in the total cells harboring both homozygous and heterozygous mutation, the actual burden of homozygous JAK2V617F mutation would be under $50 \%$.

An increase in JAK2V617F mutation is associated with higher expression of downstream target genes [10-12] and enhanced granulocyte activation [6]. Several studies have reported the relationships between the mutant allele burden and clinical phenotypes [13-14, 20]. The impact of JAK2V617F mutation burden on several clinical parameters such as WBC counts, haemoglobin concentration, platelet counts, spleen size and thrombosis - especially for thrombotic events had been demonstrated in MPN patients [13-14]. In addition, association between JAK2V617F mutation burden and thrombotic risk has also been reported [15-16].
The onset of these diseases is usually gradual and associated with elevated number of blood cells and splenomegaly. In some patients the onset is presented with thrombus and bleeding. The prevalence of overt MPN and that of JAK2V617F mutation in Korean patients with splanchnic vein thrombosis (SVT) were lower than in previous reports [17]. The association between JAK2V617F mutation burden and clinical phenotype in Chinese patients is unknown. We investigated this mutation in a cohort of 170 JAK2V617F-positive patients with PV and ET to better understand the effect of the JAK2V617F mutant allele burden on survival of Chinese patients and its relationship to disease-related complications, especially thrombosis.

\section{Patients and methods}

\section{Study population}

There were 300 patients diagnosed with PV and ET at our institute from July 2009 to December 2011, and JAK2V617F mutation was identified in 186 cases including $64 \mathrm{PV}$ and $122 \mathrm{ET}$. The mutation-positive rates was 95.5\% (64/67) in PV patients and 52.4\% $(122 / 233)$ in ET patients. Notably, only 170 specimens including $54 \mathrm{PV}$ and $116 \mathrm{ET}$ were involved in this study. All patients in this study met the World Health Organization (WHO) criteria published in 2008 for the diagnosis of MPN, and written informed consent from the subjects were obtained prior to the collection of all samples. The general characteristics of the patients are shown in Table 1 and splenomegaly was determined based on ultrasound investigations.

Table 1. Clinical characteristics of 170 patients with JAK2V617F mutation.

\begin{tabular}{|c|c|c|c|c|c|c|}
\hline & PV $(\mathrm{N}=54)$ & JAK2V617F burden (Mean, \%) & P Value & ET $(N=116)$ & JAK2V617F burden (Mean, \%) & P Value \\
\hline Age, years & & & 0.447 & & & 0.572 \\
\hline$\leq 60$ & $32(59.3 \%)$ & $41.7 \%$ & & $53(45.7 \%)$ & $16.3 \%$ & \\
\hline$>60$ & $22(40.7 \%)$ & $33.6 \%$ & & $63(54.3 \%)$ & $17.3 \%$ & \\
\hline Gender & & & $0.046^{*}$ & & & 0.455 \\
\hline M & $30(55.6 \%)$ & $31.4 \%$ & & $50(43.1 \%)$ & $15.0 \%$ & \\
\hline F & $24(44.4 \%)$ & $45.3 \%$ & & $66(56.9 \%)$ & $18.1 \%$ & \\
\hline Hemoglobin $(\mathrm{g} / \mathrm{L})$ & & & - & & & $0.023^{*}$ \\
\hline$>150$ & $54(100 \%)$ & $37.1 \%$ & & $47(40.5 \%)$ & $27.0 \%$ & \\
\hline$\leq 150$ & 0 & - & & $69(59.5 \%)$ & $14.6 \%$ & \\
\hline Median & $196(176-242)$ & & & $143(64-187)$ & & \\
\hline WBC $\left(\times 10^{9} / \mathrm{L}\right)$ & & & $0.000^{*}$ & & & $0.000^{*}$ \\
\hline$\leq 10$ & $12(22.2 \%)$ & $12.1 \%$ & & $35(30.2 \%)$ & $3.2 \%$ & \\
\hline$>10$ & $42(77.8 \%)$ & $43.7 \%$ & & $81(69.8 \%)$ & $21.9 \%$ & \\
\hline Median & 18.62(3.1-43.37) & & & $15.02(3.46-96.71)$ & & \\
\hline PLT $\left(\times 10^{9} / \mathrm{L}\right)$ & & & 0.954 & & & - \\
\hline$>300$ & $38(70.4 \%)$ & $36.8 \%$ & & $116(100 \%)$ & $16.5 \%$ & \\
\hline$\leq 300$ & $16(29.6 \%)$ & $37.6 \%$ & & 0 & - & \\
\hline Median & $382(102-941)$ & & & $872(428-2860)$ & & \\
\hline Spleen & & & 0.402 & & & $0.030^{*}$ \\
\hline Splenomegaly & $13(28,46.4 \%)$ & $46.0 \%$ & & $15(56,26.8 \%)$ & $28.7 \%$ & \\
\hline Normal spleen & $15(28,53.6 \%)$ & $39.4 \%$ & & $41(56,73.2 \%)$ & $12.5 \%$ & \\
\hline Thrombosis & & & 0.645 & & & $0.018^{*}$ \\
\hline Yes & $6(16,37.5 \%)$ & $40.8 \%$ & & $23(71,31.0 \%)$ & $22.3 \%$ & \\
\hline No & $10(16,62.5 \%)$ & $45.8 \%$ & & $48(71,69.0 \%)$ & $14.1 \%$ & \\
\hline
\end{tabular}




\section{JAK2V617F mutational analysis and methods}

Heparin anti-coagulated bone marrow was collected from patients, and then mononuclear cells were separated by Lymphocyte separation medium. Genomic DNA was extracted from mononuclear cells using DNA isolation kit (Qiagen, Courtaboeuf, France) according to the manufacturer's instructions, and stored in aliquots at $-80^{\circ} \mathrm{C}$ until further use.

The presence of JAK2V617F mutation was screened by allele specific polymerase chain reaction (PCR) by using PCR System 2700 (Applied Biosystems, Singapore, CA, USA). There were two forward primers and one reverse primer. The primer sequences of JAK2 were as follows:

Forward primer 1 (F1, wild type): 5'-ATCT ATAGTCATGCTGAAAGTAGGAGAAAG-3'.

Forward primer 2 (F2, mutation type): 5'-AGCATTTGGTTTTAAATTATGGAGTATGTT-3'.

Reverse primer (R): 5'-CTGAATAGTCCTAC AGTGTTTTCAGTTTCA-3'

The $25 \mu$ l of PCR mixture consisted of $12.5 \mu l$ of $2 \times$ Taq MasterMix (ComWin Biotech, Beijing, China), $1.2 \mu \mathrm{l}$ of $\mathrm{F} 1,1.3 \mu \mathrm{l}$ of $\mathrm{F} 2,2.5 \mu \mathrm{l}$ of $\mathrm{R}, 5.5 \mu \mathrm{l}$ of deionized water, and $2 \mu$ of samples including about 8 ng of genomic DNA. The reactive condition involved preliminary denaturation at $94^{\circ} \mathrm{C}$ for $11 \mathrm{~min}$, followed by 36 cycles of denaturation at $94^{\circ} \mathrm{C}$ for 30 s, annealing at $55^{\circ} \mathrm{C}$ for $30 \mathrm{~s}$, extension at $72^{\circ} \mathrm{C}$ for 30 s, followed by a final elongation step at $72^{\circ} \mathrm{C}$ for $6 \mathrm{~min}$, and then maintained at $10^{\circ} \mathrm{C}$. The amplified products were separated based on molecular weight by electrophoresis using a $2 \%$ agarose gel. The amplified PCR products were sequenced by using the BigDye Terminator chemistry (Applied Biosystems) and analysed on an ABI 3100 capillary sequencer.

\section{JAK2V617F mutation burden}

All 170 patients with JAK2V617F mutation were screened using allele specific PCR assay. These positive samples were detected using Real-time polymerase chain reaction (RT-PCR) with the 7500 real-time PCR system (Applied Biosystems, Hayward, CA, USA).

Forward primer: 5'-AGCTTTCTCACAAGCAT TTGGTT-3'.

Reverse primer: 5'-CAAAAACAGATGCTCT GAGAAAGG-3'.

The TaqMan MGB probe $T$ (mutation type):

5'-VIC-AATTATGGAGTATGTTTCTGTGGA-3' MGBNFQ.

The TaqMan MGB probe $G$ (wild type):

5'-FAM-TAAATTATGGAGTATGTGTCTGT-3' MGBNFQ.
The primers were purchased from Invitrogen (Invitrogen, USA) and the TaqMan MGB probes were purchased from Applied Biosystems (Hayward, USA). The RT-PCR mixture ( $25 \mu \mathrm{L}$ final volume) contained $12.5 \mu \mathrm{l}$ Platinum SuperMix-UDG (Invitrogen, USA), $0.1 \mu \mathrm{l}$ ROX, $0.5 \mu 110 \mu \mathrm{M}$ forward primer, $0.5 \mu \mathrm{l}$ $10 \mu \mathrm{M}$ reverse primer, $0.3 \mu \mathrm{l} 10 \mu \mathrm{M}$ probe $\mathrm{T}, 0.3 \mu \mathrm{l} 10 \mu \mathrm{M}$ probe G, DNA template (total for about 100ng), and deionized water. Amplification was performed by a standard protocol recommended by the manufacturer $\left(50^{\circ} \mathrm{C}\right.$ for $2 \mathrm{~min} ; 9^{\circ} \mathrm{C}$ for $10 \mathrm{~min}$; 40 repeated cycles of $95^{\circ} \mathrm{C}$ for $15 \mathrm{~s}, 55^{\circ} \mathrm{C}$ for $20 \mathrm{~s}$ and $72^{\circ} \mathrm{C}$ for $\left.1 \mathrm{~min}\right)$. The reaction of each sample was run in triplicates. The JAK2V617F proportion was calculated from cycle threshold (CT). Every sample has a value of $\Delta \mathrm{CT}$, which is the difference of the values of CT between the two probes. The ratio of JAK2V617F mutation to wildtype was $2-\Delta \mathrm{CT}$. The proportion of JAK2V617F

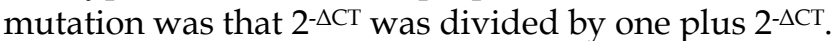

\section{Statistical analysis}

Numerical variables were tested for normal distribution with the Kolmogorov-Smirnov test. Data are expressed as mean \pm standard deviation (SD). Statistical analysis was either t-test or non-parametric Mann-Whitney-Wilcoxon U test, based on the distribution of the studied variable. The non-parametric Pearson's product-moment correlation analysis was used to test for the relationship between JAK2V617F mutation burden and different clinical variables. For normally distributed variables, Pearson correlation was used. Comparisons of categorical variables between groups were carried out using the $\chi^{2}$ test. Logistic Regression was used to test for the influence of many factors on categorical variables. Cox proportional hazard regression was used to test the impact of different covariates on risk of thrombosis in ET patients. And all tests for statistical significance were two-tailed and $P$ values less than 0.05 were considered to be statistically significant. Kaplan-Meier analysis was used to examine whether age $(<60$ years $=0$; $\geq 60$ years $=1)$, gender $($ male $=0$; female $=1)$, and JAK2V617F mutation status ( $<37.1 \%$ JAK2V617F allele burden $=0$, $\geq 37.1$ JAK2V617F allele burden $=1$ for PV; and $<16.5 \%$ JAK2V617F allele burden $=0, \geq 16.5 \%$ JAK2V617F allele burden=1 for ET) were related with PV and ET patients' survival respectively.

\section{Results}

\section{The accuracy of PCR assay in detecting JAK2V617F mutation}

JAK2V617F mutation status was screened by three primers allele specific PCR assay using genomic DNA as a template. The amplified products were 
separated by electrophoresis on a $2 \%$ agarose gel. The mutant DNA was detected using RT-PCR and confirmed by sequencing. The results were consistent between allele specific PCR and RT-PCR which confirmed that the RT-PCR method to detect the JAK2V617F mutation burden was feasible.

\section{JAK2V617F mutation status and patients' characteristics}

Table 1 summarizes clinical characteristics of all the 170 JAK2V617F positive patients $(P V=54$ and $\mathrm{ET}=116)$. Among these patients, 80 were male and 90 were female. The median age at diagnosis was 60 years (ranging from 11-89 years). Regarding leukocyte count, $47(27.7 \%)$ patients had no leukocytosis (WBC low $\left.10 \times 10^{9} / \mathrm{L}\right)$, the other $123(72.3 \%)$ had leukocytosis (WBC over $10 \times 109 / \mathrm{L}$ ). PV patients with leukocytosis (WBC above $10 \times 10^{9} / \mathrm{L}$ ) had higher JAK2V617F allele burden than the control group (WBC low $10 \times 10^{9} / \mathrm{L}$ ) $(P<0.001)$. And in PV patients, the median of WBC counts was $18.62 \times 109 / \mathrm{L}$, while it was $15.02 \times 109 / \mathrm{L}$ in ET patients $(P<0.01)$. In ET with high hemoglobin level, the mean JAK2V617F mutation burden was more than those without elevated hemoglobin level $(P<0.05)$ (Table 1). Spleen size was recorded in 84 of the 170 patients. Among them, 28 patients $(33.3 \%)$ had splenomegaly at the time of diagnosis. The proportion of splenomegaly is $46.4 \%$ in PV and $26.8 \%$ in ET ( $P$ $<0.05$ ) (Table 1). 15 ET patients with splenomegaly had significantly $(P<0.05)$ higher JAK2V617F allele burden (mean $=28.7 \%$, ranging from $0.1-67.6 \%$ ) when compared to patients without splenomegaly $($ mean $=$ $12.5 \%$, ranging from $0.1-82.3 \%)(P<0.05)$ (Table 1$)$. However, there was no difference between PV patients with or without splenomegaly $(P>0.05)$ (Table 1).

For patients with ET at diagnosis, the Pearson's product-moment correlation analysis showed a direct correlation between JAK2V617F mutant allele burden and hemoglobin concentration $(\mathrm{r}=0.395, P=0.001)$, WBC count $(r=0.453, P=0.000)$, spleen size $(r=0.321$, $P=0.001)$, and thrombosis $(r=0.421, P=0.000)$. And for patients with $\mathrm{PV}$ at diagnosis, a direct correlation was observed between JAK2V617F mutant allele burden and WBC count $(r=0.503, P=0.000)$, and gender $(r=$ $0.287, P=0.041)$.

\section{JAK2V617F mutation burden in PV and ET}

Median JAK2V617F allele burden for all patients was $15.6 \%$ (ranging from $0.1-96.2 \% ; 33.2 \%$ in PV and $7.0 \%$ in ET); JAK2V617F allele burden in PV was significantly higher than in ET $(P<0.001)$ (Figure 1$)$. Table 2 showed the proportion of JAK2V617F allele burden in PV and ET. The proportion of JAK2V617F allele burden which is more than $20 \%$ was $68.5 \%$ in
PV patients, while only $26.7 \%$ of the ET patients had such burden level (Chi-Squared test, $P<0.001$ ).

Table 2. JAK2V617F allele burden in PV and ET.

\begin{tabular}{lll}
\hline Allele burden & PV N=54 (\%) & ET N=116 (\%) \\
\hline$>50 \%$ & $19(35.2)$ & $13(11.2)$ \\
$20 \sim 50 \%$ & $18(33.3)$ & $18(15.5)$ \\
$1 \sim 20 \%$ & $11(20.4)$ & $44(37.9)$ \\
$<1 \%$ & $6(11.1)$ & $41(35.3)$ \\
\hline
\end{tabular}

JAK2V617F mutation burden in PV and ET

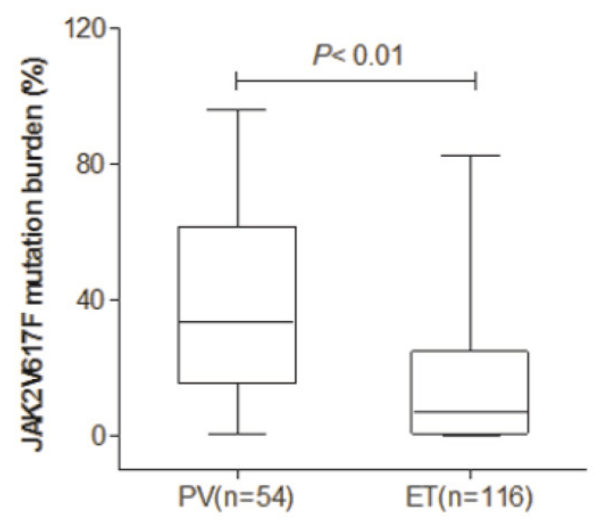

Figure 1. JAK2V617F mutation allele burden in patients with $\mathrm{PV}$ and ET. Box-plots show the proportion of JAK2V617F mutation burden in PV and ET. JAK2V617F positive population analysed with RT-PCR $(n=170)$. The difference in JAK2V617F allele burden was highly significant between the two disease entities $(P<0.01)$.

We found that the proportion of JAK2V617F allele burden in PV was higher than in ET. In addition, the WBC counts and splenomegaly events in PV and ET were compared. The median WBC count was $18.62 \times 10^{9} / \mathrm{L}$ in PV and $15.02 \times 10^{9} / \mathrm{L}$ in ET $(P<0.05)$. The proportion of splenomegaly was $46.4 \%$ in PV and $26.8 \%$ in ET $(P<0.05)$. These observations confirmed the association of JAK2V617F mutation with splenomegaly and increased WBC counts $(P<0.05)$.

\section{JAK2V617F mutation burden and clinical complications}

87 out of 170 patients had been recorded for thrombotic events. Major thrombotic events occurred in 28 patients before diagnosis $(\mathrm{PV}=6, \mathrm{ET}=22)$, including splenic infarction $(n=6)$, cerebral embolism $(n=15)$, microcirculatory disturbances in lower limbs $(n=5)$, acute myocardial infarction $(n=1)$ and pulmonary embolism $(\mathrm{n}=1)$. In addition, 2 patients $(\mathrm{PV}=1$, $\mathrm{ET}=1$ ) developed intraperitoneal hemorrhage at diagnosis. At the end of observation, all of the patients had received cytoreductive therapy. Aspirin was given to the patients with thrombocytosis. No correlation was found between JAK2V617F mutant allele 
burden and the risk of thrombosis for the whole patient cohort. However, ET patients with thrombotic events had significantly $(P<0.05$, Mann-Whitney $U$-test) higher JAK2V617F allele burden (median $=11.1 \%$, range from $0.1-70.3 \%$ ) when compared with patients without thrombosis (median $=3.8 \%$, range from $0.1-64.2 \%)(P<0.05)$. Among the 22 patients with thrombosis, 3 cases with ET had thrombotic history. We examined a retrospective analysis including age, JAK2V617F allele burden, thrombotic history, WBC counts and hemoglobin level about the risk of thrombotic events using Logistic Regression. The results showed that JAK2V617F allele burden was risk factor of thrombotic events in ET patients $(P<$ $0.01)$, while the other four were not the risk factors $(P>$ $0.05)$. And it was not found that the five factors mentioned above were the risk factors of thrombotic events in PV patients $(P>0.05)$.

\section{JAK2V617F mutation burden and survival}

Kaplan-Meier analyses were performed on the entire cohort to determine the relative prognostic values of known prognostic variables and JAK2V617F allele burden for overall survival. Date of last follow-up was 1 November 2012 or date of last contact. The median follow-up time of PV survivors was 31 months (range, 8-141 months), 31 months (range, 3-360 months) for ET survivors. All patients were scheduled to visit the doctor every 3 months and the data was documented and collected. During follow-up, no patients progressed to post-PV/ET myelofibrosis, MDS or leukemia. In PV patients, due to no dead case, we could not analyze the influence of the JAK2V617F allele burden on overall survival (OS). Among 116 ET patients, 2 (1.2\%) died and the causes of death were intraperitoneal hemorrhage in one patient and lung infection with leg dropsy in the other. Univariate analysis showed that the JAK2V617F allele burden analyzed as categorical variable, did not affect survival. And it also showed that no prognostic variable affected survival, including WBC counts, age, gender, splenomegaly and thrombosis $(P>0.05)$.

Kaplan-Meier analyses were used to analyze "event free survival" to confirm a risk of the thrombosis. It was found that thrombosis had no statistical effect on "event free survival" on ET patients $(P>0.05)$ (Figure 2). Male, high hemoglobin (HGB above $150 \mathrm{~g} / \mathrm{L}$ ), and increased JAK2V617F mutation burden (JAK2V617F allele burden $\geq 16.5 \%$ ) were the risk factors of thrombosis in patients with PV $(P<0.05)$. But these variables were not the risk factors of thrombosis for ET $(P>0.05)$. No other variables were found to be risk factors in patients with PV or ET, including WBC counts, age and splenomegaly $(P>0.05)$. Multivariable analysis (Table 3 ) showed that these covariates ana- lyzed either as a continuous or categorical variable, did not significantly correlate with the risk of thrombosis in ET patients by Cox proportional hazard regression, except for JAK2V617F allele burden.

Survival Functions

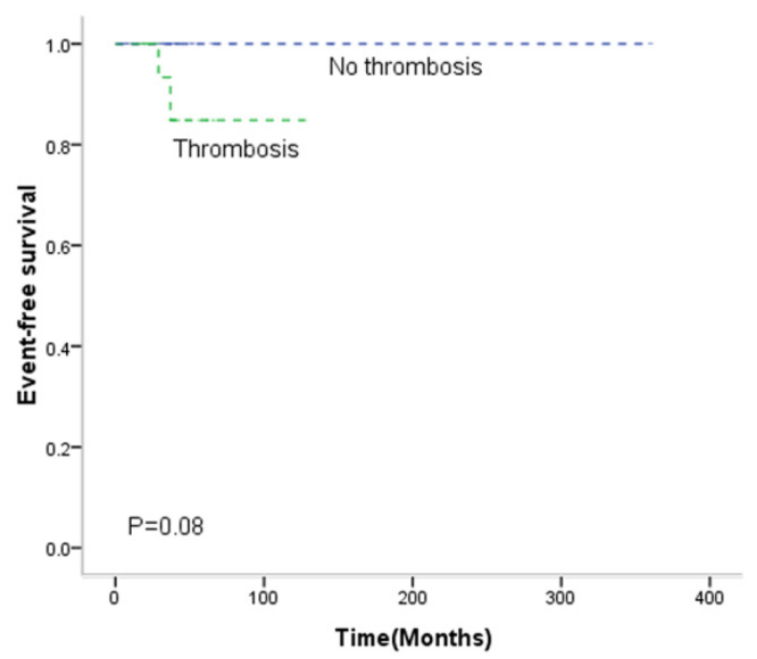

Figure 2. The effect of thrombotic events on event free survival in ET patients. Kaplan-Meier analysis for statistical significance is two-tailed and $\mathrm{P}$ values less than 0.05 are considered statistically significant.

Table 3. Multivariable analysis including clinical parameters in 71 patients with ET

\begin{tabular}{ll}
\hline Covariates & $\begin{array}{l}\text { Thrombosis (hazard ratio with 95\% confidence } \\
\text { interval) }\end{array}$ \\
\hline Age,years & $0.79(0.26-2.42) P=0.68$ \\
Gender & $2.43(0.64-9.26) P=0.19$ \\
Leukocyte count & $0.73(0.19-2.75) P=0.64$ \\
$>10 \times 109 / \mathrm{L}$ & \\
Hemoglobin level & $0.76(0.20-2.88) P=0.68$ \\
JAK2V617F allele burden & $1.23(1.08-1.39) P=0.02^{*}$ \\
Spleen size & $1.61(0.46-5.73) P=0.46$ \\
\hline
\end{tabular}

\section{Discussion}

In 2005, four groups reported the existence of an acquired clonal point mutation in JAK2 gene in MPN patients [1-4]. The JAK2V617F mutation was present in most PV patients and in substantial proportion of patients with ET. Scott et al. reported that JAK2V617F mutation did not occur in nonhematologic cancers and it was rare in myeloid malignancies other than the classic BCR/ABL-negative MPN [19]. JAK2V617F mutations had been found highly sensitive allele-specific PCR assays in approximately $95 \%$ of PV patients and $50 \%$ of ET patients [3-4]. Currently, JAK2V617F mutation is considered as a genetic diagnostic criterion for MPN.

Several studies had shown that the increase of JAK2V617F allele burden was likely to have a major 
impact on disease phenotype and was correlated with other prognostic variables in MPN patients [13, 20]. The JAK2V617F allele burden varied in MPN patients, which in part contributed to the clinical course of MPN [21]. The JAK2V617F allele burden was significantly different among patients with PV and ET [12]. Elevated JAK2V617F mutation burden could be associated with higher risk of myelofibrosis, increased haemoglobin levels and systemic symptoms. However, its effect on WBC counts, splenomegaly and thrombotic risk were controversial $[13,20]$. It had been reported that JAK2V617F mutation burden could be related with spleen size and WBC count [18]. In this study, we investigated the percentage of JAK2V617F mutated alleles in PV and ET, and compared the mutation burden among the above two diseases. We found that in Chinese patients, the JAK2V617F mutation burden was higher in PV than that in ET. The WBC counts and splenomegaly were higher in PV than in ET. Our study confirmed that the JAK2V617F mutation level was positively correlated with WBC counts and the risk of splenomegaly. Additionally, patients with elevated hemoglobin level had higher proportion of JAK2V617F mutation burden than those with normal hemoglobin level.

It has previously been suggested that gender might influence the percentage of JAK2V617F-positive disease, since it happened more frequently in men with PV [22] and women with ET [23]. It was quite intriguing that males seemed to have higher JAK2V617F allele burdens than females [8]. However, in our study, sex and age did not affect JAK2V617F allele burden in ET patients.

Thrombophilia, which severely impacts on morbidity and mortality of MPN patients, is variably characterized by microcirculatory disturbances, arterial and venous thromboses that often precede disease recognition including visual symptoms, ischaemic stroke and pulmonary embolism. A number of studies suggested leukocytosis as a novel marker for vascular risk [24, 25]. However, qualitative abnormalities of leukocytes, particularly polymorphonuclear leukocytes- the most abundant proportion of WBC, can occur in these diseases and contribute to the activation of the haemostatic system [26]. In this study, 28 patients $(\mathrm{PV}=6, \mathrm{ET}=22)$ had thrombotic events prior to or at diagnosis. However, neither WBC counts nor JAK2V617F mutation burden appeared to be a risk factor for thrombosis in patients with PV and ET in this study.

The impact of JAK2V617F mutation on thrombosis was controversial in MPN patients. It was reported that JAK2V617F mutant represented an independent factor associated with thrombosis [27]. Interestingly, JAK2V617F mutant allele burden was reported to not significantly correlate with the risk of thrombosis in PV patients [21]. And it was also not significantly correlated with thromboembolic events in ET cases [28]. In this study, no relationship was found between JAK2V617F mutation burden and the risk of thrombosis. However, ET patients with thrombotic events had significantly higher JAK2V617F mutation burden when compared to those without thrombosis. ET patients were often accompanied by thrombocytosis which could cause the thrombosis, and JAK2V617F mutation was the important mechanism of this disease. Therefore, our finding was complied with the clinical characteristics of these patients.

Previous study considered that more than $50 \%$ of JAK2V617F mutation burden was homozygous [9]. However, some patients may have both heterozygous and homozygous mutations which end up with a total JAK2V617F mutation rate below 50\%. In our study, we found that JAK2V617F mutation burden in most of the PV but not ET patients were more than $20 \%$. Thus, $20 \%$ was selected as cut off for JAK2V617F mutation burden. $68.49 \%$ of PV patients had JAK2V617F allele burden above this cutoff, whereas, only $26.7 \%$ of ET patients exhibited JAK2V617F allele burden above $20 \%$. This limit will be considered as an additional diagnostic marker when PV and ET patients have increasing complete blood cells. Further studies are needed to investigate this observation and explore the possible mechanism.

It has been reported that the JAK2V617F mutant burden did not affect survival in MPN patients with the exception of advanced aged patients [21, 29]. In this current study, we also found that JAK2V617F mutation burden did not affect survival. Moreover, we found that no other prognostic variables affected survival including age. However, it should be noted that separated mononuclear cells were used in this study, and in some cases the cell population carrying the JAK2 mutation (mostly granulocyte fraction) might be excluded. Hence, the results of our study might have been compromised in certain. In the future, we planned to optimize our experiment system and achieve the accurate results.

In summary, JAK2V617F mutation burden in PV was higher than that in ET in Chinese MPN patients. The phenotypic presentation of PV and ET, such as WBC counts, hemoglobin level, splenomegaly, and thrombosis were correlated with JAK2V617F mutation burden.

\section{Acknowledgements}

This work was supported by grants from the Jiangsu Province of China (BRA2011218 and RC2011105), National Natural Science Foundation of 
China (Grant No.81270591) and Priority Academic Program Development of the Jiangsu Higher Education Institutions (PAPD).

\section{Competing Interests}

The authors have declared that no competing interest exists.

\section{References}

1. James C, Ugo V, Le Couedic JP, Staerk J, Delhommeau F, Lacout C, Garçon L, Raslova H, Berger R, Bennaceur-Griscelli A, Villeval JL, Constantinescu SN, Casadevall N, Vainchenker W. A unique clonal JAK2 mutation leading to constitutive signaling causes polycythemai vera. Nature. 2005;434(7037):1144-1148.

2. Kralovics R, Passamonti F, Buser AS, Teo SS, Tiedt R, Passweg JR, Tichelli A, Cazzola M, Skoda RC. A gain-of-function mutation of JAK2 in myeloproliferative disorders. N Engl JMed. 2005;352(17):1779-1790.

3. Levine RL, Wadleigh M, Cools J, Ebert BL, Wernig G, Huntly BJ, Boggon TJ, Wlodarska I, Clark JJ, Moore S, Adelsperger J, Koo S, Lee JC, Gabriel S, Mercher T, D'Andrea A, Fröhling S, Döhner K, Marynen P, Vandenberghe P, Mesa RA, Tefferi A, Griffin JD, Eck MJ, Sellers WR, Meyerson M, Golub TR, Lee SJ, Gilliland DG. Activating mutation in the tyrosine kinase JAK2 in polycythemia vera, essential thrombocythemia, and myeloid metaplasia with myelofibrosis. Cancer Cell. 2005;7(4):387-397.

4. Baxter EJ, Scott LM, Campbell PJ, East C, Fourouclas N, Swanton S, Vassiliou GS, Bench AJ, Boyd EM, Curtin N, Scott MA, Erber WN, Green AR, Cancer Genome Project. Acquired mutation of the tyrosine kinase JAK2 in human myeloproliferative disorders. Lancet. 2005;365(9464):1054-1061.

5. Akada H, Yan D, Zou H, Fiering S, Hutchison RE, Mohi MG. Conditional expression of heterozygous or homozygous Jak2V617F from its endogenous promoter induces a polycythemia vera-like disease. Blood. 2010; 115(17):3589-3597.

6. Passamonti F, Rumi E, Pietra D, Della Porta MG, Boveri E, Pascutto C, Vanelli L, Arcaini L, Burcheri S, Malcovati L, Lazzarino M, Cazzola M. Relation between JAK2 (V617F) mutation status, granulocyte activation and constitutive mobilization of CD34-positive cells into peripheral blood in myeloproliferative disorders. Blood. 2006;107(9):3676-3682.

7. Kittur J, Knudson RA, Lasho TL, Finke CM, Gangat N, Wolanskyj AP, Li CY, Wu W, Ketterling RP, Pardanani A, Tefferi A. Clinical correlates of JAK2V617F allele burden in essential thrombocythemia. Cancer. 2007;109(11):2279-2284.

8. Larsen TS, Pallisgaard N, Møller MB, Hasselbalch HC. The JAK2 V617F allele burden in essential thrombocythemia, polycythemia vera and primary myelofibrosis- impact on disease phenotype. Eur J Haemotol. 2007;79(6):508-515.

9. Campbell PJ, Baxter EJ, Beer PA, Scott LM, Bench AJ, Huntly BJ, Erber WN, Kusec R, Larsen TS, Giraudier S, Le Bousse-Kerdilès MC, Griesshammer M, Reilly JT, Cheung BY, Harrison CN, Green AR. Mutation of JAK2 in the myeloproliferative disorders: timing, clonality studies, cytogenetic associations, and role in leukemic transformation. Blood. 2006;108(10):3548-3555.

10. Kralovics R, Teo SS, Buser AS, Brutsche M, Tiedt R, Tichelli A, Passamonti F, Pietra D, Cazzola M, Skoda RC. Altered gene expression in myeloproliferative disorders correlates with activation of signaling by the V617F mutation of Jak2. Blood. 2005;106(10):3374-3376.

11. Tefferi A, Sirhan S, Lasho TL, Schwager SM, Li CY, Dingli D, Wolanskyj AP, Steensma DP, Mesa R, Gilliland DG. Concomitant neutrophil JAK2 mutation screening and PRV-1 expression analysis in myeloproliferative disorders and secondary polycythaemia. Br J Haematol. 2005;131(2):166-171.

12. Lippert E, Boissinot M, Kralovics R, Girodon F, Dobo I, Praloran V, Boiret-Dupré N, Skoda RC, Hermouet S. The JAK2-V617F mutation is frequently present at diagnosis in patients with essential thrombocythemia and polycythemia vera. Blood. 2006;108(6):1865-1867.

13. Vannucchi AM, Antonioli E, Guglielmelli P, Rambaldi A, Barosi G, Marchioli R, Marfisi RM, Finazzi G, Guerini V, Fabris F, Randi ML, De Stefano V, Caberlon S, Tafuri A, Ruggeri M, Specchia G, Liso V, Rossi E, Pogliani E, Gugliotta L, Bosi A, Barbui T. Clinical profile of homozygous JAK2 617V >F mutation in patients with polycythemia vera or essential thrombocythemia. Blood. 2007;110(3):840-846.

14. Tefferi A, Strand JJ, Lasho TL, Knudson RA, Finke CM, Gangat N, Pardanani A, Hanson CA, Ketterling RP. Bone marrow JAK2V617F allele burden and clinical correlates in polycythemia vera. Leukemia. 2007;21(9):2074-2075.

15. Cheung B, Radia D, Pantelidis P, Yadegarfar G, Harrison C. The presence of the JAK2V617F mutation is associated with a higher haemoglobin and increased risk of thrombosis in essential thrombocythemia. Br J Haematol. 2006;132(2):244-245

16. Finazzi G, Rambaldi A, Guerini V, Carobbo A, Barbui T. Risk of thrombosis in patients with essential thrombocythemia and polycythemia vera according to JAK2 V617F mutation status. Haematologica. 2007:92(1):135-136.

17. Yoo EH, Jang JH, Park KJ, Gwak GY, Kim HJ, Kim SH, Kim DK. Prevalence of overt myeloproliferative neoplasms and JAK2 V617F mutation in Korean patients with splanchnic vein thrombosis. Int J Lab Hematol. 2011;33(5):471-476.
18. Barosi G, Bergamaschi G, Marchetti M, Vannucchi AM, Guglielmelli $P$, Antonioli E, Massa M, Rosti V, Campanelli R, Villani L, Viarengo G, Gattoni E, Gerli G, Specchia G, Tinelli C, Rambaldi A, Barbui T; Gruppo Italiano Malattie Ematologiche Maligne dell'Adulto (GIMEMA) Italian Registry of Myelofibrosis. JAK2 V617F mutational status predicts progression to large splenomegaly and leukemic transformation in primary myelofibrosis. Blood. 2007;110(12):4030-4036.

19. Scott LM, Campbell PJ, Baxter EJ, Todd T, Stephens P, Edkins S, Wooster R, Stratton MR, Futreal PA, Green AR. The V617F JAK2 mutation is uncommon in cancers and in myeloid malignancies other than the classic myeloproliferative disorders. Blood. 2005;106(8):2920-2921.

20. Tefferi A, Lasho TL, Schwager SM, Strand JS, Elliott M, Mesa R, Li CY, Wadleigh M, Lee SJ, Gilliland DG. The clinical phenotype of wild-type, heterozygous, and homozygous JAK2(V617F) in polycythemia vera. Cancer. 2006;106(3):631-635

21. Passamonti F, Rumi E, Pietra D, Elena C, Boveri E, Arcaini L, Roncoroni E, Astori C, Merli M, Boggi S, Pascutto C, Lazzarino M, Cazzola M. A prospective study of 338 patients with polycythemia vera: the impact of JAK2 (V617F) allele burden and leukocytosis on fibrotic or leukemic disease transformation and vascular complications. Leukemia. 2010;24(9):1574-1579.

22. Passamonti F, Rumi E, Pungolino E, Malabarba L, Bertazzoni P, Valentini M, Orlandi E, Arcaini L, Brusamolino E, Pascutto C, Cazzola M, Morra E, Lazzarino M. Life expectancy and prognostic factors for survival in patients with polycythemia vera and essential thrombocythemia. Am J Med. 2004;117(10):755-761.

23. Campbell PJ, Scott LM, Buck G, Wheatley K, East CL, Marsden JT, Duffy A, Boyd EM, Bench AJ, Scott MA, Vassiliou GS, Milligan DW, Smith SR, Erber WN, Bareford D, Wilkins BS, Reilly JT, Harrison CN, Green AR; United Kingdom Myeloproliferative Disorders Study Group; Medical Research Council Adult Leukaemia Working Party; Australasian Leukaemia and Lymphoma Group. Definition of subtypes of essential thrombocythaemia and relation to polycythaemia vera based on JAK2 V617F mutation status: a prospective study. Lancet. 2005;366(9501):1945-1953.

24. Carobbio A, Antonioli E, Guglielmelli P, Vannucchi AM, Delaini F, Guerini V, Finazzi G, Rambaldi A, Barbui T. Leukocytosis and risk stratification assessment in essential thrombocythemia. J Clin Oncol. 2008;26(16):2732-2736.

25. Landolfi R, Di Gennaro L, Barbui T, De Stefano V, Finazzi G, Marfisi R, Tognoni G, Marchioli R; European Collaboration on Low-Dose Aspirin in Polycythemia Vera (ECLAP). Leukocytosis as a major thrombotic risk factor in patients with Polycythemia Vera. Blood. 2007;109(6):2446-2452.

26. Landolfi R, Di Gennaro L, Falanga A. Thrombosis in myeloproliferative disorders: pathogenetic facts and speculation. Leukemia. 2008;22(11):2020-2028.

27. Vannucchi AM. JAK2 mutation and thrombosis in the myeloproliferative neoplasms. Curr Hematol Malig Rep. 2010; 5(1):22-28.

28. Wang J, Xu Z, Liu L, Gale RP, Cross NC, Jones AV, Qin T, Ai X, Xu J, Zhang T, Sun X, Li Q, Zhang P, Zhang Y, Xiao Z. JAK2V617F allele burden, JAK2 46/1 haplotype and clinical features of Chinese with myeloproliferative neoplasms. Leukemia. 2013; 27(8):1763-1767.

29. Helbig G, Wieczorkiewicz A, Woźniczka K, Wiśniewska-Piąty K, Rusek A, Kyrcz-Krzemień S. The JAK2V617F tyrosine kinase mutation has no impact on overall survival and the risk of leukemic transformation in myelofibrosis. Med Oncol. 2012; 29(4):2379-2384. 